\section{Value of third sputum smear for detection of pulmonary tuberculosis in HIV infected patients}

\author{
Majid Marjani, Payam Tabarsi, \\ Parvaneh Baghaei, Davoud Mansouri, \\ Mohammad Reza Masjedi, \\ Ali Akbar Velayati \\ Clinical Tuberculosis and Epidemiology \\ Research Center, National Research \\ Institute of Tuberculosis and Lung \\ Disease, Masih Daneshvari Hospital, \\ Shahid Beheshti University of Medical \\ Sciences, Tehran, Iran
}

\section{Abstract}

We evaluated diagnostic yield of third sputum smear in patients co infected with HIV for detection of pulmonary tuberculosis (TB). Among 139 pulmonary tuberculosis cases confirmed with positive sputum culture, diagnostic yield of first smear of sputum with acid fast staining was $81.9 \%$. Incremental yield of $2^{\text {nd }}$ and $3^{\text {rd }}$ samples was $11.7 \%$ and $6.3 \%$ respectively. So two sputum smears may be enough for primary evaluation of HIV infected patients suspected to TB.

\section{Introduction}

Tuberculosis (TB) is known as the leading cause of death of patients with HIV infection. TB resulted in 200,000 deaths among 700,000 cases of TB-HIV co infection during the year 2008. ${ }^{1}$

Despite recent advances in early diagnosis of tuberculosis, microscopic examination of the sputum smear remains the most appropriate method particularly in the majority of countries which are endemic for disease. Number of sputum samples to be examined for screening of TB remains one of the most frequent questions. ${ }^{2}$

Due to the lower numbers of acid fast bacilli (AFB) in respiratory secretions of HIV positive patients, the sensitivity of microscopic sputum analysis may be reduced. ${ }^{3}$ Negative sputum smear pulmonary TB is more common in HIV positive patients and has higher mortality rate.

World Health Organization (WHO) advised examination of two sputum specimens instead of three sputum specimens in HIV infected patients with clinical suspicion of TB before marking it as a smear negative case. ${ }^{4}$ This rec- ommendation was based on the studies which contained very few HIV infected patients. So, although strongly supported by expert opinion, there are significant evidence gaps. ${ }^{5}$

Reducing the numbers of specimen examined, can help saving time and money especially in resource limited setting so more cases will be detected. ${ }^{6}$

Thus, we performed this study to evaluate sensitivity of two versus three sputum smears for diagnosis of pulmonary tuberculosis in patients with HIV coinfection.

\section{Materials and Methods}

We conducted a cross-sectional study at our center, National Research Institute of Tuberculosis and Lung Disease, NRITLD, Tehran, Iran. We retrospectively reviewed the microbiological laboratory records of HIV infected patients diagnosed as a new case of pulmonary tuberculosis from 2006 to 2009 . The patients who were 18 years old or more have been enrolled. The patients were asked to give morning sputum samples for three consecutive days if possible. All of the obtained specimens were investigated by Ziehl-Neelsen staining (concentration method and light microscope) and mycobacterial culture on conventional Lowenstein-Jensen media. The tests were done at the WHO-approved national mycobacteriologic reference laboratory.

Definite diagnosis of pulmonary TB was based on a positive culture for Mycobacterium tuberculosis. Patients whom were diagnosed by other ways such as mycobacterial examination of bronchoalveolar lavage (BAL) were excluded. Results of first, second and third sputum were assessed. A sputum smear positive case was defined as a positive result in at least one of specimens. For calculation of incremental yield and increase in sensitivity, the definitions similar to the work of Mase $e t$ al. were used..$^{5}$ Sensitivity was calculated in comparison to culture as gold standard. For this purpose the number of patients who had three specimens examined by smear and culture, of which at least one was culture-positive, was used as denominator. For calculation of sensitivity of first sputum smear, number of patients with first specimen positive was used as numerator. To calculate the increase of sensitivity about $2^{\text {nd }}$ sputum sample, number of patients with first specimen negative and second positive and about $3^{\text {rd }}$ sputum sample number of patients with first two sputum negative and third specimen positive was used.

The incremental yield of the second specimen was calculated as the number of patients with first specimen negative and second smear positive divided by the number of smear positive patients who had three specimens exam-
Correspondence: Majid Marjani, Clinical Tuberculosis and Epidemiology Research Center. National Research Institute of Tuberculosis and Lung Disease, Masih Daneshvari Hospital, Shahid Beheshti University of Medical Sciences, Tehran, Iran.

Tel/Fax: +98.21.2610.9590.

E-mail:marjani@nritld.ac.ir

Key words: tuberculosis, HIV, diagnosis, pulmonary infection, sputum.

Contributions: MM, design, gathering data, writing of paper; $\mathrm{PT}$, design, gathering data; $\mathrm{PB}$, gathering data, analysis; DM, writing of paper; MRM, design, writing of paper; AAV, design.

Conflict of interests: the authors report no potential conflict of interests.

Received for publication: 15 0ctober 2011.

Revision received: 14 June 2012.

Accepted for publication: 13 May 2012.

This work is licensed under a Creative Commons Attribution NonCommercial 3.0 License (CC BYNC 3.0).

(C) Copyright M. Marjani et al., 2012

Licensee PAGEPress, Italy

Infectious Disease Reports 2012; 4:e35

doi:10.4081/idr.2012.e35

ined. The incremental yield of the third specimen was calculated as the number of patients with first two specimens negative and the third smear was positive divided by the number of smear positive patients who had three specimens examined.

\section{Results}

During the period of study, 150 patients with HIV and pulmonary TB were diagnosed by Mycobacteriologic diagnostic examination. Twenty seven of them had extra pulmonary TB concomitantly. Most of them were men (148 persons, $98.6 \%)$. Mean of age was 40 and CD4 cell count ranged from 2 to 700 (mean=87.5, median=42). We excluded 2 cases which were diagnosed by positive smear and culture of BAL specimen and also 9 more cases with negative culture of sputum in spite of positive smear(s). Among 139 cases with culture positive pulmonary TB, 133 had at least one positive sputum smear for AFB and 116 of them had three specimens examined. Sensitivity of the first sputum sample for acid fast staining was $75 \%$ in culture proven cases who had three specimens examined by smear and culture ( 87 of 116). Increase in sensitivity was $11.2 \%$ (13 of 116 ) and $5.1 \%$ (6 of 116) for $2^{\text {nd }}$ and $3^{\text {rd }}$ sam- 
ples. Diagnostic yield of first AFB smear of sputum was $83.4 \%$. Incremental yield of $2^{\text {nd }}$ and $3^{\text {rd }}$ samples was $11.7 \%$ and $6.3 \%$ respectively. It means diagnostic yield for first two sputum samples is $93.7 \%$. Results are summarized in Table 1.

\section{Discussion}

This study demonstrates that two sputum smears may be enough for primary evaluation of HIV infected patients suspected to TB. Diagnostic yield of $3^{\text {rd }}$ sputum is very low (only $6.3 \%)$, so it is not necessary for $93.7 \%$ of patients. It is prudent to examine third sputum sample and more if first two sputum samples were negative for acid fast staining and suspicion to TB is high, for example in the presence of abnormal CXR without any known reason. Reducing the number of specimens examined from three to two may be more important in setting with limited resources.

Our findings are consistent with few previous studies demonstrating low diagnostic yield of $3^{\text {rd }}$ sputum sample in HIV infected patients TB suspects. ${ }^{2,7-9}$

In a study was performed by Matee and colleagues, among 103 culture positive cases of TB HIV co infection, incremental diagnostic yield for sputum microscopy was $92.1 \%, 1.8 \%$ and $7.1 \%$ for first, second and third specimens, respectively. ${ }^{?}$

In other similar studies the incremental diagnostic yield of $3^{\text {rd }}$ sputum was $2 \%, 1.4 \%$ and $0.8 \%{ }^{8-10}$ Noeske and co-workers compared sensitivity of two versus three sputum smears among culture positive TB patients co-infected with HIV. It was $76 \%$ and $73 \%$ respectively without any statistically significant difference. ${ }^{2}$

In our study, percentage of smear positive TB cases was higher than others. It may be due to some methodological differences concerning sputum collection and staining techniques among studies. For example fluorochrome technique was used in some studies, ${ }^{9,10}$ but others performed light microscopy and acid fast staining. We used concentrated method and Ziehl-Neelsen staining. Previous studies were based on using combination of one or two spot sputum samples and one or two first morning samples. We asked patients to provide three first morning sputum samples. In spite of these methodological diversions, results were similar.

Because mycobacterial culture is very effective in diagnosing of tuberculosis among HIV positive patients, especially smear negative cases, some authors investigated the yield of the culture of $3^{\text {rd }}$ sputum sample. Monkongdee and colleges found that Mycobacterium tuber-

Table 1. Diagnostic yield of acid fast bacilli smear examination among pulmonary tuberculosis cases co infected with HIV.

\begin{tabular}{lcc} 
Sputum specimen & Smear sensitivity & Incremental diagnostic yield \\
First sample & $75 \%$ & $81.9 \%$ \\
Second sample & $11.2 \%$ & $11.7 \%$ \\
\hline Third sample & $5.1 \%$ & $6.3 \%$ \\
\hline
\end{tabular}

culosis can be detected by culture in brothbased medium in $71 \%$ of HIV-TB co infected patients and an additional $17 \%$ and $10 \%$ cases were diagnosed by $2^{\text {nd }}$ and 3 rd samples. ${ }^{8}$ Similar results reported by Cain et al. They found that the best diagnostic approach is to examine minimally two and ideally three samples for culture in liquid media. ${ }^{11}$ It is important to consider that culture is not available in many resource limited setting and the sensitivity of solid media is lower than broth media. ${ }^{8}$

This study had some limitations. Our center is referral for tuberculosis patients in Iran. So our cases may be more complicated and their disease may be more advanced, although we enrolled only new cases with suspicious to TB. On the other hand, sputum examinations were performed by national reference laboratory. But in the field, the quality of sputum smear microscopy depends on the performance of local laboratories. So it is necessary to consider WHO recommendation about external quality assurance systems under the supervision of the national reference laboratories. ${ }^{12}$

\section{Conclusions}

In conclusion, because diagnostic yield of third sputum is low, two sputum samples is enough for microscopic examination in HIV infected patients suspected to TB. If suspicion is high in spite of two negative sputum smears, more sputum specimens or performing other diagnostic techniques are prudent.

\section{References}

1. World Health Organization. Global tuberculosis control: surveillance, planning, financing: WHO report 2008. 2008; Geneva: WHO.

2. Noeske J, Dopico E, Torrea G, et al. Two vs. three sputum samples for microscopic detection of tuberculosis in a high HIV prevalence population. Int J Tuberc Lung Dis 2009;13:842-7.

3. American Thoracic Society. Diagnostic standards and classification of tuberculosis in adults and children. Am J Respir Crit Care Med 2000;161:1376-95.

4. World Health Organization. Improving the diagnosis and treatment of smear-negative pulmonary and extrapulmonary tuberculosis among adults and adolescents: recommendations for HIV-prevalent and resource-constrained settings. 2006; Geneva: WHO.

5. Mase S, Ramsay A, Ng N, et al. Yield of serial sputum specimen examinations in the diagnosis of pulmonary tuberculosis: a systematic review. Int $\mathrm{J}$ Tuberc Lung Dis 2007;11:485-95.

6. Walker D, McNerney R, Mwembo MK, et al. An incremental cost-effectiveness analysis of the first, second and third sputum examination in the diagnosis of pulmonary tuberculosis. Int J Tuberc Lung Dis 2000;4:246-51.

7. Matee M, Mtei L, Lounasvaara T, et al. Sputum microscopy for the diagnosis of HIV-associated pulmonary tuberculosis in Tanzania. BMC Public Health 2008;8:68.

8. Monkongdee P, McCarthy KD, Cain KP, et al. Yield of acid-fast smear and mycobacterial culture for tuberculosis diagnosis in people with human immunodeficiency virus. Am J Respir Crit Care Med 2009; 180:903-8.

9. Leonard MK, Osterholt D, Kourbatova EV, et al. How many sputum specimens are necessary to diagnose pulmonary tuberculosis? Am J Infect Control 2005;33:58-61.

10. Crampin AC, Floyd S, Mwaungulu F, et al. Comparison of two versus three smears in identifying culture-positive tuberculosis patients in a rural African setting with high HIV prevalence. Int J Tuberc Lung Dis 2001;5:994-9.

11. Cain KP, McCarthy KD, Heilig CM, et al. An algorithm for tuberculosis screening and diagnosis in people with HIV. N Engl $\mathrm{J}$ Med. 2010;362:707-16.

12. World Health Organization. Early detection of tuberculosis: an overview of approaches, guidelines and tools. Available from: http://whqlibdoc.who.int/hq/ 2011/WHO_HTM_STB_PSI_2011.21_eng. pdf. Accessed on: March 2012. 